\title{
A Study on Sentiment Analysis: Methods and Tools
}

\author{
Abhishek Kaushik ${ }^{1}$, Anchal Kaushik ${ }^{2}$, Sudhanshu Naithani ${ }^{3}$ \\ ${ }^{1}$ Kiel University of Applied Sciences, Computer and Electrical Department, Sokratesplatz, 1, 24149 Kiel, Germany \\ ${ }^{2}$ Amity University, Department of Management Studies, Sector 125, Noida, India \\ ${ }^{3}$ Kurukshetra University, Department of Computer Science, Thanesar Taluk, Kurukshetra, India
}

\begin{abstract}
The purpose of social media has created many chances for people to publicly voice their beliefs, simply when they are employed to deliver an opinion hit a vital problem. Sentiment Analysis is a case of natural language processing which could mark the mood of the people about any specific product by analysis. Sentiment Analysis is a process of automatic extraction of features by mode of notions of others about specific product, services or experience. The Sentiment Analysis tool is to function on a series of expressions for a given item based on the quality and features. Sentiment analysis is also called Opinion mining due to the significant volume of opinion. Analyzing customer opinion is very important to rate the product. To automate rate the opinions in the form of unstructured data is been a challenging problem today. Thus, this paper discusses about Sentiment analysis methods and tools used.
\end{abstract}

Keywords: Data Mining, Opinion Mining, Opinion Summarization, Sentiment Analysis, Text Mining, Web Mining.

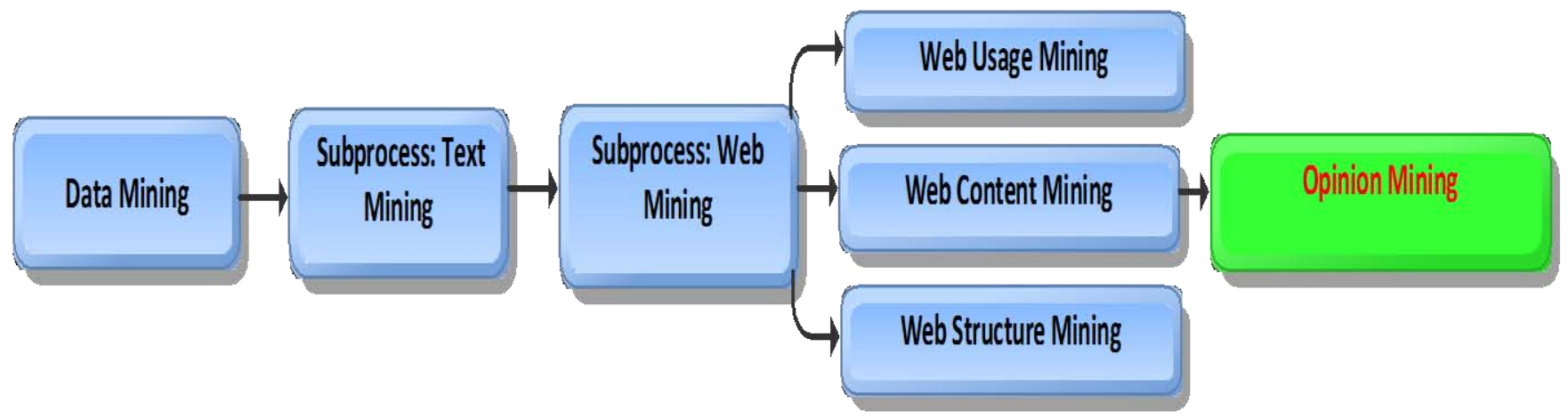

Figure 1: Hierarchy of Data Mining

\section{Introduction}

The era of electronic information in every phase of life is evolving rapidly, which tends to produce a large number of data. As an outcome huge volumes of data are generated in field of technology, business, healthcare, tourism, emarketing, etc. Automated analysis systems are meant for analysis, summarization and classification of data and number of efficient methods to store huge amount of data. Text mining is an approach used different fields like machine learning, information retrieval, statistics, and computational linguistics for opinion mining. Web mining is a subset of text mining used to mine the unstructured web data in the form Content mining, Web Structure mining and Web Usage mining. The aim of sentiment analysis is to make an automated machine able to recognize and categorize emotions [2]. A thought, view, or attitude based on emotion instead of reason is called sentiment. Figure 1 shows the different sub level of Data Mining and the branches of sentiment analysis.

\section{Literature Overview}

Bakhtawar Seerat et al [15] proposed the method of opinions extraction from an online web page and the limitation of Sentiment analysis. Meena Rambocas [20] concluded all the challenges marketers can face when using sentiment analysis as an alternative technique capable of triangulating qualitative and quantitative methods through innovative real time data collection and analysis. G.Vinodhini et al [10] proposed an Overview of different opinion mining techniques. Blessy Selvam et al [3] proposed different approaches of sentiment classification and the existing methods with the framework. Rudy Prabowo [16] formed a new approach by combining rule-based classification, supervised learning and machine learning and tested it on movie reviews, product reviews and MySpace comments. And also proposed a semi automatic approach to get better effectiveness. Archana Shukla [19] introduced a tool to tell the quality of the document or its usefulness based on the annotations. Ayesha Rashid et al [1] presented the limitations on different sentiment level and the methods used in sentiment analysis. Dongjoo Lee et al [4] proposed to use the PMI method to use for large corpus to achieve higher accuracy. Dr.Ritu Sindhu et al [14] presented different levels of analysis and issues in sentiment analysis. S.Chandrakala et al [5] proposed a work on recent papers on sentiment analysis and its related tasks with future challenges. Bo Pang [17] gave a new machine learning method that determines sentiment polarity. Arti Buche et al [11] proposed the Naive Bayes algorithm and also Hidden Markov Model to calculate the Entropy and Purity measure in string mining. S.Padmaja et al [6] proposed a work on Machine Learning Models for text classification. . Nile M. Shrike et al [13] compared the

\section{Volume 4 Issue 12, December 2015}




\section{International Journal of Science and Research (IJSR) \\ ISSN (Online): 2319-7064}

Index Copernicus Value (2013): 6.14 | Impact Factor (2014): 5.611

accuracy using Bayes, Maximum Entropy and Support Vector Machine. Raisa Varghese et al [7] proposed the structure of sentiment analysis. Vijay B . Roth et al [9] have compared the synopsis of different approaches used for sentiment analysis. Nidhi Mishra et al [12] proposed the inner view of sentiment analysis at different levels David Osimo et al [2] proposed an outline for a new Research Challenge on Sentiment Analysis. Sindhu, Chandrakala et al [8] proposed a systematic flow and Machine learning approaches to optimize the performance. Alec Go [18] proposed a novel approach to classify sentiment of the twitter message automatically and showed that machine learning algorithms (Naive Bayes, Maximum Entropy, and SVM) have accuracies above $80 \%$ when trained with emoticon data.

\section{Information Source}

User view is an important factor for the improvement of the quality of services. Blogs, review sites, data and micro blogs provide a good information of the products and services provided to clients.

Blogs: The name relates all the blog sites is called blogosphere [1]. People express about their thoughts they want to share with others on a blog. Blog pages [1] have become the popular platform to share ones personal views about specific products

Review sites: The opinions of others is being an important factor while purchasing anything. A large number of users express their views on a particular product. These reviews are easily available on the Internet. The re-viewer's data used in most of the opinion classification gather from the ecommerce websites [10] like www.flipkart.com .

Data Set: The dataset contains different types of product reviews (including Books, DVDs, Electronics and Kitchen appliances) and movie reviews extracted from Flip-kart and IMDB webpage.

\section{Sentiment Analysis}

Sentiment analysis is a technique which is used to extract the meaningful information in the documents [6]. In general, opinion mining tries to figure out the sentiment of a writer about some specific aspect and also the overall contextual polarity of a document. The sentiment may be a judgment, mood or evaluation of the writer [2]. A core issue in this field is an opinion classification, where a review is classified as a positive or negative evaluation of a subjected object (film, book, etc.). The assessment of sentiment can be done in two ways:

4.1 Direct opinions: It gives positive or negative sentiment about the product directly [12]. For example, "The food quality of this hotel is poor" expresses a direct opinion.

4.2 Comparison: It means to compare the subject with any other similar objects [12]. For example, "The food quality of the hotel-a is better than that of hotel-b." expresses a comparison. Figure 2 had a workflow of Opinion Mining.
The views are being extracted from writers review over their comment. Opinion feature extraction is a sub-process of opinion mining [15]. Pre-processing In this process, raw data taken and is pre-processed for feature extraction.

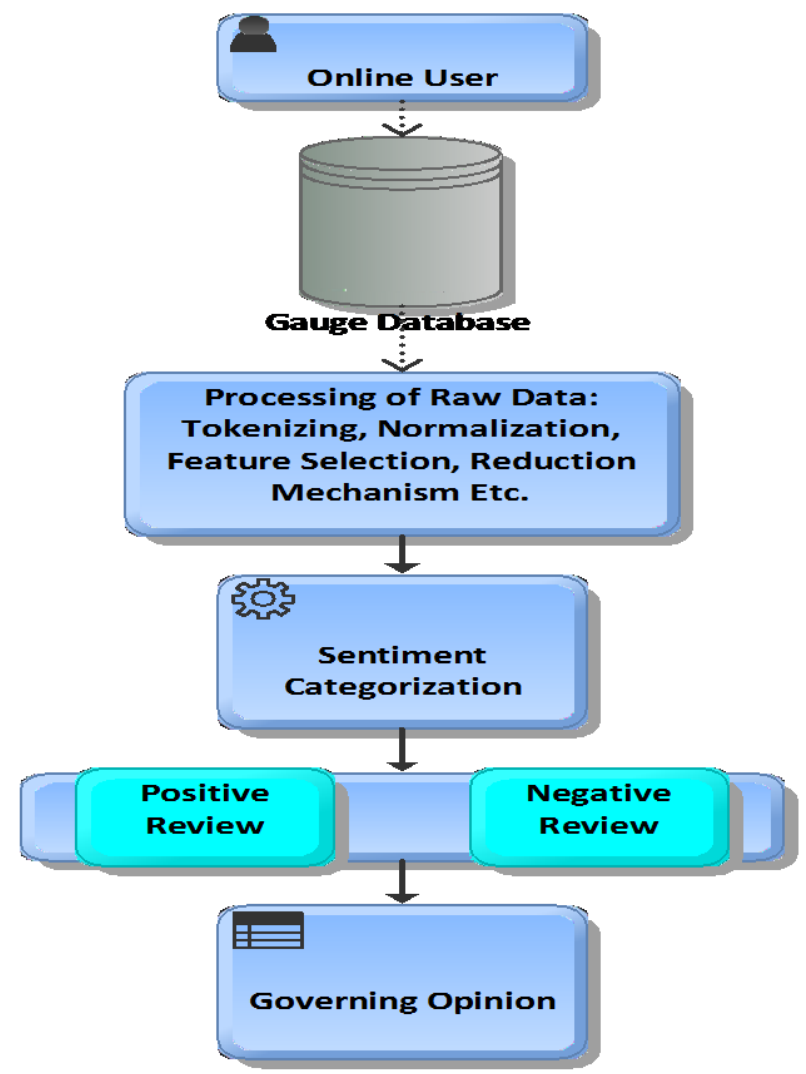

Figure 2: Work flow of Opinion Mining

The preprocessing phase [1] has been further divided into a number of sub phases as follows:

Tokenization is the process to split up into tokens by removing white spaces, commas and other symbols, etc. Stop word Removal removes words (like ,a, an, the, of, for,). Stemming reduce the relevant tokens into a single type. Normalization is a process that has English texts to be published in both higher and lower case characters and turns the entire document or sentences into lowercase/uppercase.

Feature extraction phase deals with feature types [3] (which identifies the type of features used for opinion mining), feature selection (used to select good features for opinion classification), feature weighting mechanism (weights each feature for good recommendation) reduction mechanisms (features for optimizing the classification process).

Types of features used for opinion mining could be:

1) Term frequency (The presence of the term in a document carries a weight age).

2)Term co-occurrence (features which occurs together like uni-gram, bi-gram or n-gram),

3)Part of speech information (POS tagger is used to separate POS tokens).

4)Opinion words (Opinion words are words which express positive (good) or negative (bad) emotions) [3].

5)Negations (Negation words (not, not only) shift sentiment 


\section{International Journal of Science and Research (IJSR) \\ ISSN (Online): 2319-7064}

Index Copernicus Value (2013): 6.14 | Impact Factor (2014): 5.611

orientation in a sentence)

6) Syntactic dependency (It is represented as a parse tree and it contains word dependency based features)

\section{Feature Selection}

1)Information gain (based on the presence and absence of a term in a document a threshold is set and the terms with less information gain is removed).

2)Odd Ratio (It is suitable for binary class domain where it has one positive and one negative class for classification.

3)Document Frequency measures the number of appearances of a term in the available number of documents in the corpus and based on the threshold computed the terms are removed. Features weighting mechanism The mechanisms are of two types. They are 1: Term Presence and Term Frequency- word which occurs occasionally contains more information than frequently occurring words. 2: Term frequency and inverse document frequency (TFIDF) Documents are rated where highest rating is given to words that appear regularly in a few documents and lowest rating for words that appear regularly in every document. Feature Reduction Feature reduction reduces the feature vector size to optimize the performance of a classifier.

Reduction of the number of features in the feature vector can be done in two different ways in which top n-features can be left in the vector and either low level or unwanted linguistic features could be removed. Adjectives only Adjectives have been used most frequently as features amongst all parts of speech. A strong correlation between adjectives and subjectivity has been found. Although all the parts of speech are important people most commonly used adjectives to depict most of the sentiments and a high accuracy have been reported by all the works concentrating on only adjectives for features generation. Adjective-Adverb Combination Most of the adverbs have no prior polarity.

But when they occur with sentiment bearing adjectives, they can play a major role in determining the sentiment of a sentence. Adverbs alter the sentimental value of the adjective that they are used with. Adverbs of degree, on the basis of the extent to which they modify this sentimental value, are classified as:

- Adverbs of affirmation: certainly, totally

- Adverbs of doubt: maybe, probably

- Strongly intensifying adverbs: exceedingly, immensely

- Weakly intensifying adverbs: barely, slightly

- Negation and minimizers: never Some of the positive

Adjectives are as follows dazzling, brilliant, phenomenal, excellent and fantastic. Negative Adjectives: suck, terrible, awful, unwatchable, hideous.

\section{Standard Structure of Sentimental Analysis}

Opinion Mining also called sentiment analysis is a process of finding user's opinion towards a topic or a product. Opinion mining concludes whether the user's view is positive, minus, or neutral about a product, issue, event, etc. Opinion mining and summarization process involve three primary steps, first is Opinion Retrieval, Opinion Classification and Opinion
Summarization. Review Text is retrieved from review websites. Opinion text in blog, reviews, comments, etc. contains subjective information about the topic.

Reviews classified as positive or negative review. Opinion summary is generated based on features opinion sentences by considering frequent features about a matter.

\subsection{Opinion Retrieval}

It is the procedure of collecting review text from review sites. Different review websites contain reviews for products, movies, hotels and news.

\subsection{Information retrieval}

Techniques such as web crawler can be employed to collect the review text data from many sources and store them in a database. This step involves retrieval of reviews, micro-blogs and comments by user.

\subsection{Opinion Classification}

Primary steps in sentiment analysis are a classification of review text. Given a review document $\mathrm{M}=\{\mathrm{M} 1 \ldots \mathrm{M} 1\}$ and a predefined category set $\mathrm{K}=$ \{positive, negative $\}$, sentiment classification is to classify each day in $M$, with a label expressed in $\mathrm{K}$. The approach involves classifying review text into two forms namely positive and negative [9]. Machine learning and dictionary based approach is more popular [3].

\subsection{Opinion Summarization}

Summarization of opinion is a major character in the opinion mining process. Summary of reviews provided should be based on features or subtopics that are mentioned in the reviews. Many works have been done on summarization of product reviews [9].

The opinion summarization process mainly involves the following two approaches. Feature based summarization a type summarization involves the finding of frequent terms (features) that are appearing in many reviews. The summary is submitted by selecting sentences that contain particular feature information. Characteristics present in review text can be identified using Latent Semantic Analysis (LSA) method.

Term frequency is a count of term occurrences in a document. If a term has higher frequency it means that the condition is more import for summary presentation. In many product reviews certain product features come out frequently and associated with user opinions about it. Fig. 3 has the architecture of Opinion Mining which says how the input is being classified on the various steps to summarize the reviews.

\section{Volume 4 Issue 12, December 2015}




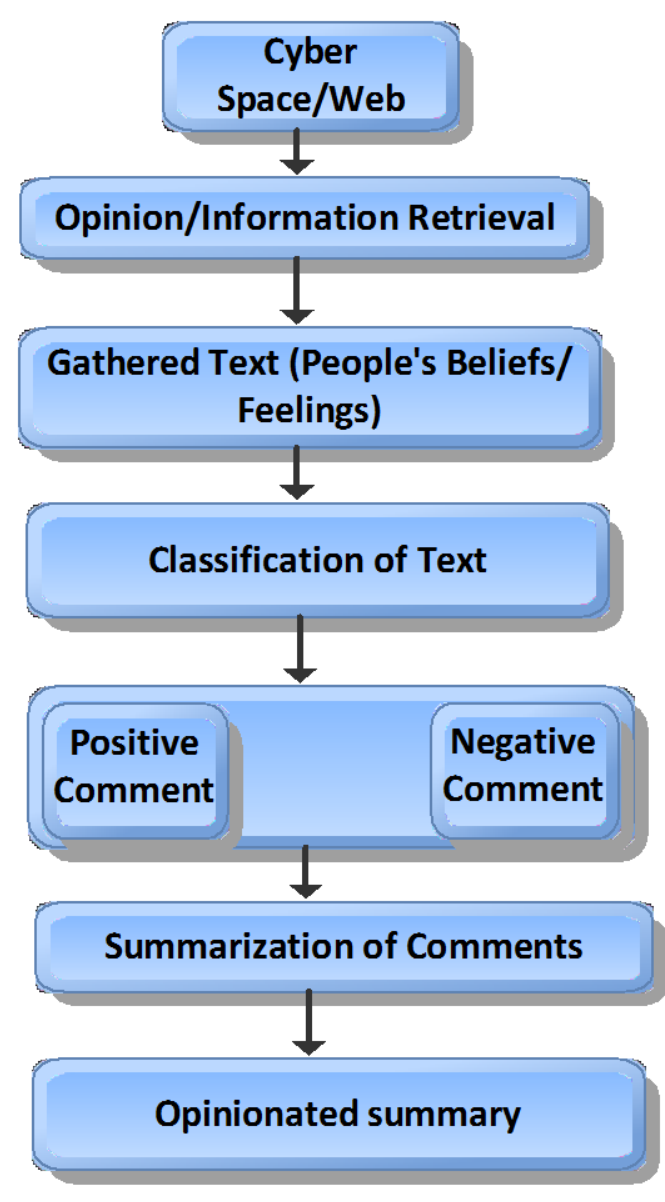

Figure 3: Architecture of opinion mining

\section{Hierarchy of Opinion Mining}

- Document level Opinion Mining- A single document of opinionated text works as a basic data unit in this level [7]. Here the document level classification is a single review about a topic is viewed. Merely in the forums or blog scenario, there are possibilities for comparative sentences to appear and for clients to compare one product with another that has alike characteristics and that's how document level analysis is not suitable for forums and web logs. Therefore the subjectivity/objectivity arrangement is very vital in this type of classification.

- Sentence level Opinion Mining- The calculated polarity of each sentence is considered in the case of sentence level Opinion Mining. The same classification approach as applied in document level, can be reactive to the sentence level classification problem also, but Objective and subjective sentences [12] necessarily be localized. Opinion words are carried by subjective sentences. These sentiment words aid to determine the sentiments related to that entity. After which the polarity classification takes place into positive and negative classes.

- Phrase level Opinion Mining- This level of classification is much more pinpointed approach to opinion mining. Here phrases containing opinion words are observed and the phrase level class is completed. Only in some special cases, where contextual polarity also matters, the effect may not be fully precise.

\section{Techniques}

Major data mining techniques used to dig the knowledge and information are: generalization, classification, clustering, genetic algorithm, association rule mining, data visualization, neural networks, fuzzy logic, Bayesian networks, and, decision tree. Number 5 has the techniques of Opinion Mining. Figure 5. Techniques of Opinion Mining

- Supervised Machine Learning: Classification is most oftenly used and very popular data mining technique [11]. Classification used to divide the possible results from a given data set is based on the basis of a defined set of attributes and a given predictive attributes. The given dataset is used as the training dataset consist of independent variables (properties of the dataset) and a dependent attributes (predicted attribute). A training dataset created model test on text corpus holds the same attributes but no predicted attribute. Accuracy of model checks on how faultless it is making a prediction. Double Propagation Algorithm is used to extract Product features and sentenced words.

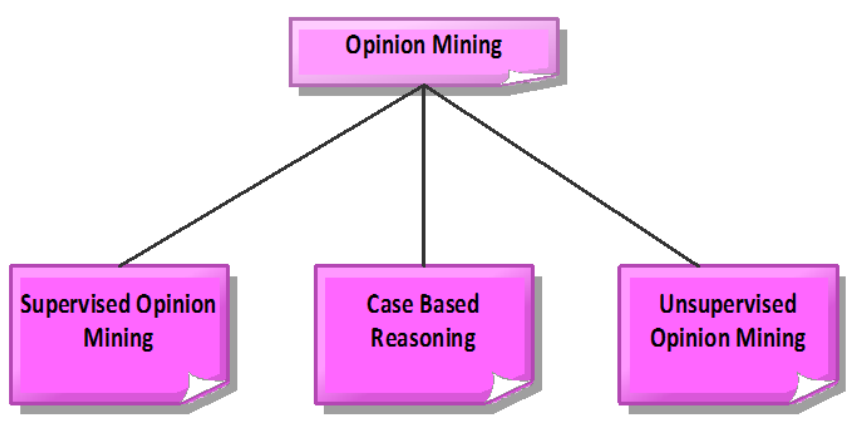

Figure 4: Techniques of Opinion

- Unsupervised Learning- It differs to supervised learning, because unsupervised learning does not have definite targeted output connected with the input. Class label for any instance is not known so this technique of learning is about to learn by observation. Clustering is a technique which is also used in unsupervised learning. Clustering is an approach of bunching objects with similar properties into a group. Objects in a cluster are always dissimilar to the objects in other clusters.

- Case Based Reasoning- Case based reasoning is one of the emerging Artificial Intelligence supervised techniques. $\mathrm{CBR}$ is a fierce tool of computer reasoning and crack the problems (cases) in the closest way to real time scenario. This is a problem solving technique in which knowledge is personified as past cases in the library and it is not dependent on classical rules. The solutions of all the cases are stored in CBR warehouse known as Knowledge base or Case base.

\section{Semantic Orientation}

Problem of Opinion mining can be divided into two parts which are sentiment classification [13] and feature based opinion mining. The trouble of taking out the semantic orientation (SO) of a text (i.e., whether the text is positive or negative towards a peculiar subject matter) often takes as a 


\section{International Journal of Science and Research (IJSR) \\ ISSN (Online): 2319-7064}

Index Copernicus Value (2013): 6.14 | Impact Factor (2014): 5.611

starting point the problem of determining semantic orientation for individual speech. The hypothesis is that, if the SO of relevant words in a text is given, SO for the entire text can be determined. The SO approach to Sentiment analysis is an unsupervised learning because it does not need advance training in order to mine the data. Figure 6 shows the details of the classification of approaches of semantic orientation

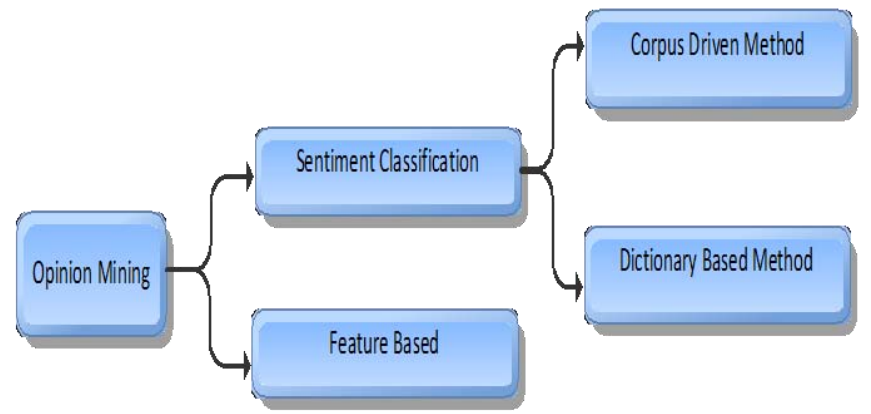

Figure5: Classification of Approaches of Semantic Orientation

- Corpus Based Approach- Emotional affinity of words is determined by Popular corpus-driven method. Emotional affinity is meant to learn their probabilistic affective scores from large corpora. The method to assign a happiness factor to words depending on the frequency of their occurrences in happy-labelled blog posts compared to their total frequency in a corpus containing blog posts labelled with "happy" and "sad" mood annotations. They also compare the happiness factor scores of words with the scores in the list.

- Dictionary Based Approach- Dictionary based approach contains used lexical resources (e.g-Word Net) which work as an asset to automatically acquire emotion-related words for emotion classification experiments. They start from a set of primary emotion adjectives, and then retrieve alike words from Word Net by utilizing all senses of all words in the synsets that contain the emotion adjectives. The process takes advantage of the synonym and hyponym relations in Word Net to manually find alike words to nominal emotion words. The affective weights are automatically acquired from a very large text corpus in an unsupervised fashion.

\section{Tools Used In Opinion Mining}

The tools used in the process of tracking the opinion or polarity from the user's generated contents are:

- Review Seer tool - Work done by aggregation sites is automated by this tool. To collect positive and negative opinions for assigning a score to the extracted feature terms, the Naive Bayes classifier approach is used. The results are displayed as a simple opinion sentence [10].

- Web Fountain - Beginning definite Base Noun Phrase (BNP) heuristic approach is used here for extracting the product features. Development of a simple web interface is also possible.

- Red Opal -This tool allows the users to determine the features based opinion orientations of products. It assigns the scores to each and every product based on features extracted from the customer reviews. The results are displayed by a web based interface [1].

- Opinion observer-This is an opinion mining system which is used to analyze and compare different opinions [5] on the cyber space by using user generated contents. This system illustrates the results in a graph format clearly showing opinion of the product feature by feature. It uses a WordNet Exploring method to assign prior polarity.

\section{Conclusion}

Opinion mining is an emerging sphere of data mining used to receive the knowledge of the huge mass of data (data may be customer comments, feedback and reviews on whatever product or topic etc). Much research has been carried on to mine the opinions in the contour of a document, sentence and feature level sentiment analysis. It has been examined that now the opinion mining trend is proceeding to the sentimental reviews of twitter data, comments used in Facebook on pictures, videos or Facebook status. Therefore, this paper discusses about an overview of the sentimental analysis approach of Opinion Mining in detail with the techniques and tools.

\section{References}

[1] Ayesha Rashid et al, "A Survey Paper: Areas, Techniques and Challenges of Opinion Mining", International Journal of Computer Science (IJCSI), Vol 10 Issue 6 No 2, Nov 2013.

[2] David Osimo and Francesco Mureddu, "Research Challenge on Opinion Mining and Sentiment Analysis".

[3] Bluesy Selvam, A. Abirami, "A Survey on Opinion Mining Framework", International Journal of Advanced Research in Computer and Communication Engineering,Vol 2, Issue 9, Sep 2013Pg No 3544-3549.

[4] Dongjoo Lee et al, "Opinion Mining of Customer Feedback Data on the Web". Seoul National University.

[5] S. Chandrakala, C. Sindhu, "Opinion Mining and Sentiment Classification: A Survey”,ICTACT Journal on Soft Computing, Oct 2012 Vol 3 Issue 1,Pg No 420-425.

[6] S.Padmaja et al, "Opinion Mining and Sentiment Analysis - An Assessment of Peoples' Belief: A Survey", International Journal of Ad hoc, Sensor \& Ubiquitous Computing IJASUC, Vol 4 No 1, Feb 2013.

[7] Raisa Varghese, Jayasree, "A Survey on Sentiment Analysis and Opinion Mining", International Journal of Research in Engineering and Technology (IJRET), Vol 2 Issue 11 Nov 2013.

[8] Sindhu, Chandrakala, "A Survey on Opinion Mining and Sentiment Polarity Classification", International Journal of Emerging Technology and Advanced Engineering.Vol 3 Issue 1, Jan 2013.

[9] Vijay. B. Roth et al, "Survey on Opinion Mining and Summarization of User Reviews on Web", International Journal of Computer Science and Information Technologies (IJCSIT), Vol 5(2), 2014. 1026-1030.

[10] G. Vinodhini et al, "Sentiment Analysis and Opinion Mining: A Survey", International Journal of Advanced Research in Computer Science and Software Engineering (IJARCSSE), Vol 2, Issue 6, June 2012.

\section{Volume 4 Issue 12, December 2015}


[11] Arti Buche, Dr.M.B.Chandak, Akshay Zadgoanakar "Opinion Mining and Analysis: A Survey", International Journal on Natural Language Computing (IJNLC) Vol 2 No 3 June 2013Pg No 39-48.

[12] Nidhi Mishra et al, "Classification of Opinion Mining Techniques", International Journal of Computer Applications, Vol 56, No 13, Oct 2012Pg No 1-6.

[13]Nilesh M. Shrike et al, "Survey of Techniques for Opinion Mining", International Journal of Computer Applications, Vol 57 No 13. Nov 2012Pg No 30-35.

[14] Dr. Ritu Sindhu, Ravendra Ratan Singh Jandail, Rakesh Ranjan Kumar, "A Novel Approach for Sentiment Analysis and Opinion Mining", International Journal of Emerging Technology and Advanced Engineering (IJETAE), Vol 4, Issue 4, April 2014.

[15] Bakhtawar Seerat, Farouque Azam, "Opinion Mining: Issues and Challenges (A Survey)", International Journal of Computer Applications, Vol49 No 9 July 2012Pg No 42-51.

[16] Rudy Prabowo, Mike Thelwell, "Sentiment Analysis: A Combined Approach".

[17] Bo Pang, Lillian Lee, "A Sentimental Education: Sentiment Analysis Using Subjectivity Summarization Based on Minimum Cuts".

[18] Alec Go, Richa Bhayani, Lei Huang, "Twitter Sentiment Classification Using Distant Supervision".

[19] Archana Shukla, "Sentiment Analtsis of Document Based on Annotation".

[20] Meena Rambocas, Joao Gama, "Marketing Research: The Role of Sentiment Analysis".

\section{Author Profiles}

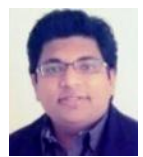

Abhishek Kaushik is currently working in Siemens, Germany as a Master thesis student. He is in the final phase of completing his Masters degree in Information Technology from Kiel University of Applied Sciences. Before starting his Masters he received his Bachelor's of Technology in Computer Science Engineering from Kurukshetra University in 2012.

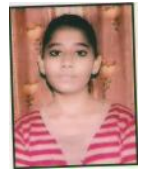

Anchal Kaushik is pursuing her MBA in Competitive Intelligence and Strategy Management from Amity University Noida. Before this she received her Bachelor's of Commerce Degree in 2014 from CCS Meerut

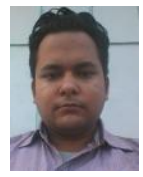

Sudhanshu Naithani has received his Bachelor's of Technology in Computer Science Engineering from Kurukshetra University in 2015. He is currently working as a research assistant under Assistant Professor Ravinder Madan at Manav Bharti University,

Solan. 